\title{
Mortalidade por armas de fogo no estado do Rio de Janeiro, Brasil: uma análise espacial
}

\author{
Célia Landman Szwarcwald ${ }^{1}$ e Euclides Ayres de Castilho ${ }^{1}$
}

RESUMO O aumento da mortalidade por armas de fogo no estado do Rio de Janeiro, Brasil, tem tomado um aspecto alarmante. $O$ objetivo deste estudo foi caracterizar a evolução temporal da mortalidade por armas de fogo neste estado, no período de 1979 a 1992, conforme sexo, idade e região de residência (capital, cinturão metropolitano e interior do estado) e estudar a propagação da epidemia ao longo do tempo e do espaço, com a utilização de técnicas de análise estatística espacial.

No período analisado, a mortalidade por armas de fogo teve o seu maior crescimento entre adolescentes de 15 a 19 anos, do sexo masculino; estas taxas variaram de 13 a 16\% para este grupo, conforme a região de residência. Para o grupo das crianças de 10 a 14 anos, a mortalidade por armas de fogo teve um acréscimo de $10 \%$ ao ano. Foi nítida a interiorização da mortalidade por armas de fogo. No início da série, observou-se uma direção preferencial de disseminação, ao longo dos municípios situados na costa leste do estado, acompanhando o trajeto de uma rodovia federal. Entre 1990 e 1992, entretanto, a difusão ocorreu em praticamente todas as direções. A constatação empírica da expansão generalizada da mortalidade por armas de fogo nega as afirmações corriqueiras de concentração da violência nos bolsões de pobreza das metrópoles brasileiras. Os programas para prevenir e controlar a epidemia devem abordar o problema sob diferentes aspectos, enfocando questões tanto no plano coletivo (proliferação de armas entre a população vinculada ao contrabando internacional de armas, aumento da criminalidade, expansão do tráfico de drogas e exclusão de oportunidades sociais), assim como no plano individual (relações e interações dos jovens com seu ambiente, em nível da família, da escola e da sociedade).

A mortalidade por causas externas tem tomado cada vez mais vulto no Brasil. No início da década de 80, a mortalidade por causas externas passou a ser a segunda principal categoria de causas de óbitos, só sendo sobrepujada pelas doenças do aparelho circulatório. Entre as causas externas, os

\footnotetext{
1 Fundação Oswaldo Cruz, Departamento de Informações para a Saúde. Correspondência e pedidos de separatas devem ser enviados a Célia Landman Szwarcwald no seguinte endereço: Departamento de Informações para a Saúde, Fundação Oswaldo Cruz, Avenida Brasil, 4365, CEP 21045-900, Rio de Janeiro, RJ, Brasil. Correio eletrônico: celia@ malaria.procc.fiocruz.br
}

homicídios apresentam a maior taxa de crescimento (1).

Um dos principais exemplos, no país, do aumento da mortalidade intencionalmente infligida é o estado do Rio de Janeiro. Em 1991, este estado tinha uma população aproximada de 13 milhões de habitantes; mais de 70\% da população viviam na região metropolitana. O Rio de Janeiro exibe, atualmente, uma das maiores taxas de mortalidade por violência, em nível nacional e internacional (2).

As causas externas têm chamado a atenção pelo seu caráter devastador entre adolescentes e adultos jovens.
Em 1990, foram responsáveis por mais de $30 \%$ dos anos potenciais de vida perdidos entre 1 e 70 anos no estado do Rio de Janeiro (3). Entre as mortes por causas externas, destacam-se as mortes por armas de fogo. Mais de 6000 vidas são perdidas, no estado, a cada ano, devido às armas de fogo. Mais da metade das mortes ocorrem entre indivíduos com menos de 30 anos (4).

Na medida em que a violência assume características de epidemia, sua prevenção tem sido, cada vez mais, tratada como prioridade na área de saúde pública (5). Adicionalmente, o 
aspecto alarmante da situação tem despertado o interesse da sociedade. $\mathrm{O}$ tema da violência, assim como as campanhas de desarmamento, vêm ganhando espaço nos meios de comunicação de massa.

No sentido de contribuir para a caracterização epidemiológica deste agravo de saúde, o presente trabalho teve como objetivo estudar a mortalidade por armas de fogo no estado do Rio de Janeiro, no período de 1979 a 1992. Após analisar a evolução temporal por sexo, idade e região de residência, uma nova dimensão foi introduzida na investigação, o componente espacial. Através de técnicas de análise estatística espacial (6), os autores identificaram as áreas de maior risco e as direções preferenciais de dependência no espaço, buscando-se estabelecer os caminhos de propagação da violência no estado do Rio de Janeiro ao longo do tempo.

\section{MATERIAIS E MÉTODOS}

Para a realização deste trabalho foram utilizadas as informações relativas a óbitos do Sistema de Informações sobre Mortalidade do Ministério da Saúde (SIM/MS), para o período de 1979 a 1992 (7). Este período foi escolhido para o estudo já que 1979 e 1992 correspondem, respectivamente, ao primeiro e ao último ano com disponibilidade de informações provenientes do SIM/MS.

Os denominadores das taxas de mortalidade foram calculados por interpolação e projeção geométrica a partir das populações referidas nos censos demográficos de 1980 e $1991(8,9)$.

Para constituir a categoria de óbitos por armas de fogo foram considerados todos os óbitos por causas externas provocados por armas de fogo, isto é, classificados segundo a nona revisão da Classificação Internacional de Doenças (CID) como "acidentes provocados por armas de fogo" (CID 922), "suicídios por arma de fogo" (CID 955), "homicídios com armas de fogo" (CID 965), "lesões por intervenção legal com armas de fogo" (CID 970), ou como "lesões por armas de fogo ignoradas se acidental ou intencionalmente infligidas" (CID 985).

Na primeira etapa, o estudo consistiu em analisar as taxas de mortalidade por armas de fogo, no período de 1979 a 1992, segundo sexo, grupo etário (10 a 14 anos; 15 a 19 anos; 20 a 24 anos; 25 a 29 anos; e 30 a 39 anos), e região de residência, ou seja, uma das três regiões em que foi subdividido o estado do Rio de Janeiro: o município da capital; o cinturão metropolitano, constituído por 12 municípios que, conjuntamente com a capital, em 1980, formavam a denominada Região Metropolitana (8); e o interior, composto pelos demais municípios (figura 1).

O comportamento temporal das taxas de mortalidade foi avaliado através da taxa anual de crescimento geométrico, expressa em percentual, estimada por meio do ajuste destas a uma regressão exponencial. A evolução no tempo da categoria das armas de fogo foi comparada à evolução apresentada pelas demais causas externas e por todas as outras causas (que não as externas).

Em etapa posterior, para caracterizar a realização espacial da mortalidade por armas de fogo no território do estado do Rio de Janeiro, foram utilizadas técnicas de análise estatística espacial, sendo consideradas como unidades geográficas os 66 municípios que compunham o estado em grande parte do período estudado, de 1979 a $1986 .{ }^{2}$ Para minimizar o efeito dos denominadores pequenos em alguns municípios, as taxas municipais de mortalidade por armas de fogo foram estimadas por taxas médias trienais, em quatro períodos distintos (1980 a 1982; 1983 a 1985; 1986 a 1988; 1989 a 1991). Para estabilizar a variância das taxas, utilizou-se a transformação da raiz quadrada, considerando-se como objeto da análise espacial a raiz quadrada das taxas médias trienais.

O conjunto de dados espaciais foi complementado pelas coordenadas geográficas (longitude e latitude) dos

\footnotetext{
2 A partir de 1987, até o início da década de 90, foram criados muitos municípios novos, sendo um pouco diferente a configuração atual.
}

centros demográficos dos municípios e por informações sobre adjacência entre eles.

Para a visualização da propagação espacial da mortalidade por armas de fogo, foram usadas técnicas de geoprocessamento, através da elaboração de mapas temáticos pelo programa MapInfo (10), considerando-se a configuração geográfica de 66 municípios, vigente de 1979 a 1986.

A análise espacial foi baseada na metodologia proposta por Cliff e Ord (6). Os municípios adjacentes foram definidos como "vizinhos"; as ponderações binárias foram atribuídas aos pares de unidades geográficas. Como medida de autocorrelação no espaço, utilizouse o coeficiente $c$ de Geary (6), conforme fórmula a seguir:

$$
c=\frac{\sum_{i}^{n} \sum_{j}^{n} w_{i j}\left(z_{i}-z_{j}\right)^{2} / \sum_{i} \sum_{j} w_{i j}}{\sum_{i}\left(z_{i}-\bar{z}\right)^{2} /(n-1)}
$$

onde $w_{i j}=1$, se as localidades $i$ e $j$ são vizinhas, e $w_{i j}=0$, caso contrário; $z_{i}=$ observação na localidade $i ; \bar{z}=\frac{1}{n} \sum_{i}^{n} z_{i}$; $n$ é o número de localidades.

No caso de independência espacial, este coeficiente aproxima-se de 1 . Por outro lado, quanto maior a dependência espacial, tanto mais o índice $c$ se aproxima de zero.

Sob a suposição de distribuição normal do processo espacial, o teste de significância da autocorrelação espacial foi baseado no valor padronizado de $c$, $(c-1) / \sqrt{ } \operatorname{var}(c)$, onde $\operatorname{var}(c)$ representa a variância de $c$, que tem distribuição assintótica normal sob a hipótese nula de independência espacial (6).

Para verificar a existência de direções preferenciais de dependência espacial, foram estudadas oito diferentes direções $(0, \pi / 8,2 \pi / 8, \ldots . ., 7 \pi / 8$ radiano), com uma tolerância bilateral de $\pi / 16$ radianos, considerando-se como origem dos eixos cartesianos o ponto situado na extremidade inferior esquerda do estado. Para esta parte da análise, seguiu-se a mesma metodologia utilizada para a detecção de autocorrelação espacial, porém foram defi- 


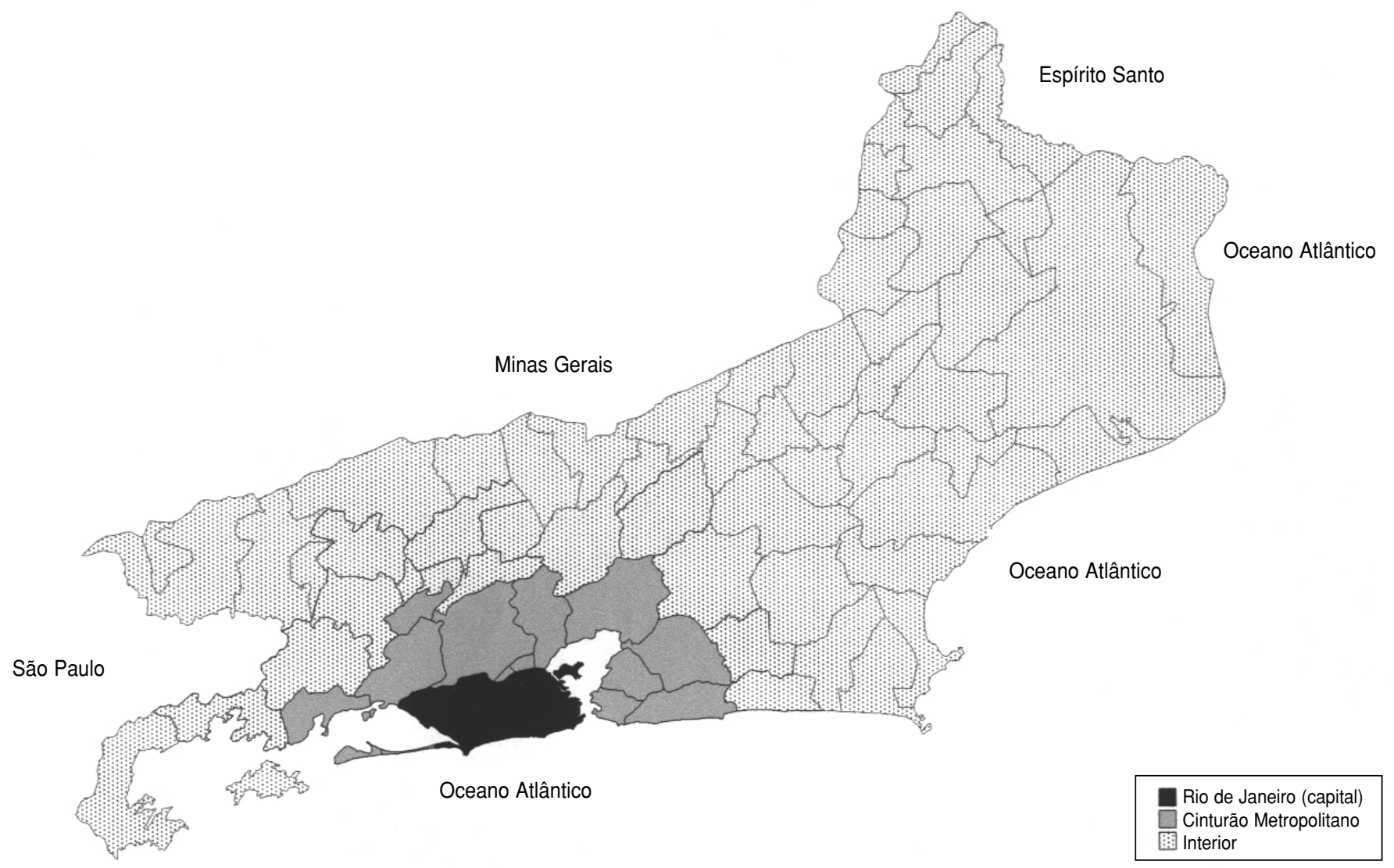

nidos como "vizinhos" os municípios adjacentes cujo eixo de ligação entre os seus centros demográficos estivesse na direção sob investigação.

\section{RESULTADOS}

\section{Panorama epidemiológico}

Entre 1979 e 1992, a taxa de mortalidade por causas externas no estado do Rio de Janeiro cresceu de 96 para 125 por 100000 habitantes. Este aumento foi provocado pelas lesões por armas de fogo, que representaram mais de $40 \%$ das mortes por causas externas (tabela 1). Enquanto a mortalidade por armas de fogo teve uma taxa de crescimento de $10 \%$ ao ano, os coeficientes relativos às mortes por demais causas externas e por todas as outras causas demonstraram estabilidade no pe- ríodo analisado. As taxas anuais de mortalidade por armas de fogo indicam que o maior acréscimo ocorreu entre 1979 e 1989, quando estas taxas atingiram o valor máximo. Nos anos subseqüentes, as taxas de mortalidade por armas de fogo passaram a ter comportamento de estabilidade.

Os dados contidos na tabela 2 revelam um grande diferença entre homens e mulheres: o índice médio de sobre-mortalidade masculina foi igual a 17,5 nos 14 anos estudados, ou seja, em média, a mortalidade masculina por armas de fogo foi 17,5 vezes maior do que a feminina. Entretanto, as taxas anuais de crescimento foram bastante próximas, indicando que o acréscimo no número de mortes por armas de fogo atingiu ambos os sexos.

O acompanhamento temporal da mortalidade proporcional por armas de fogo, segundo grupo de idade, pode ser apreciado na tabela 3. A partir de 1988, as armas de fogo passaram a ser a principal causa de morte entre os adolescentes (15 a 19 anos) e entre os adultos jovens (20 a 24 anos), explicando mais de $45 \%$ das mortes nestes grupos etários. Com taxa anual de aclive de $14 \%$, o grupo de 15 a 19 anos é o mais afetado, seguido dos adultos jovens e das crianças de 10 a 14 anos, que apresentaram aumento de $10 \%$ ao ano (tabela 4).

Os indicadores calculados por região de residência mostram a interiorização do fenômeno no estado (tabela 5). O interior do estado apresentava, no início do período, taxa de 14 por 100000 habitantes, o que representava $65 \%$ da taxa na região metropolitana. Já nos anos 90, o coeficiente alcançou o valor de 60 por 100000 , um patamar igual ou superior ao das outras regiões. 
TABELA 1. Taxas de mortalidade (por 100000 habitantes) por armas de fogo, outras causas externas, demais causas e taxas de variação anual correspondentes (\%), estado do Rio de Janeiro, Brasil, 1979 a 1992

\begin{tabular}{ccccr}
\hline Ano & $\begin{array}{c}\text { Armas } \\
\text { de fogo }\end{array}$ & $\begin{array}{c}\text { Outras causas } \\
\text { externas }\end{array}$ & $\begin{array}{c}\text { Demais } \\
\text { causas }\end{array}$ & $\begin{array}{r}\text { Total de } \\
\text { causas }\end{array}$ \\
\hline 1979 & 19,7 & 76,3 & 723,4 & 819,4 \\
1980 & 23,3 & 75,3 & 722,0 & 820,6 \\
1981 & 21,2 & 72,6 & 713,5 & 807,3 \\
1982 & 19,0 & 71,0 & 685,6 & 775,6 \\
1983 & 23,7 & 72,2 & 704,5 & 800,5 \\
1984 & 30,3 & 75,1 & 709,7 & 815,2 \\
1985 & 34,5 & 74,4 & 706,8 & 815,6 \\
1986 & 38,6 & 80,9 & 713,7 & 833,2 \\
1987 & 42,0 & 75,1 & 711,1 & 828,2 \\
1988 & 47,1 & 77,7 & 744,6 & 869,4 \\
1989 & 59,0 & 78,7 & 717,8 & 855,5 \\
1990 & 55,9 & 78,1 & 717,8 & 851,8 \\
1991 & 53,1 & 72,8 & 696,3 & 822,6 \\
1992 & 55,6 & 69,0 & 697,2 & 821,8 \\
Taxa de variação anual (\%) & $+10,1^{\mathrm{a}}$ & 0,0 & 0,0 & 0,4 \\
\hline
\end{tabular}

a Significativo em nível de $5 \%$.

A mortalidade por armas de fogo teve seu maior crescimento entre adolescentes de 15 a 19 anos de idade, do sexo masculino, residentes no interior do estado (tabela 6). Para estes, a taxa de mortalidade por armas de fogo quadruplicou e aumentou em 16\% ao ano.

TABELA 2. Taxas de mortalidade (por 100000 habitantes) por armas de fogo segundo sexo e taxas de variação anual (\%), estado do Rio de Janeiro, Brasil, 1979 a 1992

\begin{tabular}{ccc}
\hline Ano & Homens & Mulheres \\
\hline 1979 & 38,0 & 2,2 \\
1980 & 44,8 & 2,7 \\
1981 & 41,0 & 2,3 \\
1982 & 36,3 & 2,6 \\
1983 & 46,0 & 2,6 \\
1984 & 59,1 & 3,1 \\
1985 & 67,2 & 3,5 \\
1986 & 74,8 & 4,5 \\
1987 & 82,5 & 4,0 \\
1988 & 92,1 & 4,8 \\
1989 & 115,2 & 6,5 \\
1990 & 108,8 & 6,5 \\
1991 & 103,5 & 6,3 \\
1992 & 108,6 & 6,3 \\
Taxa de variação & & \\
anual (\%) & $10,2^{\mathrm{a}}$ & $9,9^{\mathrm{a}}$ \\
\hline
\end{tabular}

a Significativo em nível de 5\%.

\section{A interiorização do processo: análise espacial}

A disseminação da mortalidade por armas de fogo no estado do Rio de Janeiro pode ser melhor compreendida pela apreciação da seqüência de mapas temáticos, correspondentes, respectivamente, aos quatro períodos trienais analisados (figura 2a, 2b, 2c e 2d). A observação dos mapas mostra claramente a interiorização do processo. No começo da série, os valores elevados de mortalidade por armas de fogo se concentravam na região metropolitana; ao final do período, vários municípios localizados no interior também apresentavam taxas elevadas de mortalidade por armas de fogo.

Em todos os períodos, a autocorrelação espacial foi altamente significativa, indicando que municípios vizinhos tendem a ter menor variabilidade nas taxas por armas de fogo do que os municípios que não são adjacentes (tabela 7).

A investigação de direção na dependência espacial mostrou que a direção oeste-leste (0 radiano) foi importante em todos os anos, assim como a direção de $\pi / 8$ radiano, com exceção do período de 1983 a 1985, conforme pode ser verificado pelos valores significativos encontrados para o coeficiente de Geary correspondentes a estas duas direções (tabela 7). Estes resultados indicam uma alta concentração de taxas elevadas na região metropolitana, composta de municípios localizados ao redor da capital e cujos centros demográficos são ligados por eixos que se situam, em geral, em direções aproximadamente paralelas à direção de 0 radiano.

A direção de $\pi / 4$ radiano também se destacou como direção preferencial para a dependência espacial. As medidas de autocorrelação no espaço, traduzidas pelo coeficiente $c$ de Geary, foram significativas em nível de 5\% para os períodos de 1983 a 1985; 1986 a 1988; e 1990 a 1992. Sendo assim, além da concentração de taxas elevadas na região metropolitana, que permanece até o final da série analisada, o fenômeno parece ter se disseminado na direção de 45 graus, atingindo, a cada período de tempo, um novo grupo de municípios. É interessante notar que esta direção preferencial de difusão do processo acompanhou o litoral leste fluminense, na mesma direção da rodovia federal paralela à costa que liga Niterói (região metropolitana) a Vitória, capital do estado do Espírito Santo (figura 3).

Note-se, ainda, que os valores da autocorrelação onidirecional diminuem expressivamente nos dois últimos períodos, ou seja, no final da década de 80 os valores dos coeficientes tornaram-se menores e mais homogêneos em todas as direções (e não mais em apenas uma direção), mostrando a expansão generalizada do fenômeno.

\section{DISCUSSÃO}

No início dos anos 90, a taxa de mortalidade por causas externas no estado do Rio de Janeiro ultrapassava o valor de 120 por 100000 habitantes, um coeficiente aproximadamente igual ao dobro do coeficiente para todo o Brasil e para a América Latina. Atualmente, a taxa de mortalidade por causas externas no Rio de Janeiro se encontra no 
TABELA 3. Mortalidade proporcional (\%) por armas de fogo, outras causas externas e demais causas por grupo de idade, estado do Rio de Janeiro, Brasil, 1979 a 1992

\begin{tabular}{|c|c|c|c|c|c|c|c|c|c|}
\hline \multirow[b]{2}{*}{ Ano } & \multicolumn{3}{|c|}{10 a 14 anos } & \multicolumn{3}{|c|}{15 a 19 anos } & \multicolumn{3}{|c|}{20 a 24 anos } \\
\hline & $\begin{array}{l}\text { Armas } \\
\text { de fogo }\end{array}$ & $\begin{array}{l}\text { causas } \\
\text { externas }\end{array}$ & $\begin{array}{l}\text { Demais } \\
\text { causas }\end{array}$ & $\begin{array}{l}\text { Armas } \\
\text { de fogo }\end{array}$ & $\begin{array}{l}\text { causas } \\
\text { externas }\end{array}$ & $\begin{array}{l}\text { Demais } \\
\text { causas }\end{array}$ & $\begin{array}{l}\text { Armas } \\
\text { de fogo }\end{array}$ & $\begin{array}{l}\text { causas } \\
\text { externas }\end{array}$ & $\begin{array}{l}\text { Demais } \\
\text { causas }\end{array}$ \\
\hline 1979 & 3,2 & 41,3 & 55,5 & 17,7 & 43,4 & 38,9 & 21,7 & 43,3 & 35,0 \\
\hline 1980 & 3,7 & 39,3 & 57,0 & 21,1 & 42,1 & 36,8 & 26,0 & 39,9 & 34,1 \\
\hline 1981 & 3,4 & 41,5 & 55,1 & 18,0 & 44,6 & 37,4 & 23,1 & 40,8 & 36,1 \\
\hline 1984 & 4,2 & 42,9 & 52,9 & 25,6 & 40,5 & 33,9 & 30,5 & 40,1 & 29,4 \\
\hline 1985 & 4,0 & 43,1 & 52,9 & 32,2 & 35,9 & 31,9 & 35,1 & 37,2 & 27,7 \\
\hline 1986 & 6,1 & 45,4 & 48,5 & 35,4 & 41,4 & 33,0 & 35,8 & 36,7 & 27,5 \\
\hline 1987 & 7,8 & 37,3 & 54,8 & 36,5 & 33,4 & 30,1 & 38,9 & 33,9 & 27,2 \\
\hline 1988 & 6,8 & 44,5 & 48,7 & 41,9 & 32,2 & 25,8 & 41,8 & 32,1 & 26,2 \\
\hline 1989 & 10,5 & 39,5 & 50,1 & 46,8 & 31,7 & 21,6 & 46,8 & 29,8 & 23,4 \\
\hline 1990 & 10,1 & 43,8 & 46,1 & 46,9 & 31,1 & 22,1 & 45,5 & 31,5 & 23,0 \\
\hline
\end{tabular}

mesmo patamar de alguns poucos países da região das Américas, como a Colômbia, que também apresenta taxa superior a 100 por 100000 habitantes (11).

Em relação à mortalidade por armas de fogo, a situação é ainda mais grave. Em 1991, os Estados Unidos apresentavam taxa de mortalidade por armas de fogo de 15 por 100000 habitantes. O valor máximo encontrado, de 26 mor- tes por 100000 habitantes, nos estados de Louisiana e Nevada (12), correspondeu à metade do coeficiente no estado do Rio de Janeiro para o mesmo ano. O contraste é maior em comparação com os países europeus. Nestes países, as taxas de mortalidade por homicídios são menores do que 5 por 100000 habitantes (13).

A discussão sobre como controlar a epidemia das mortes por armas de

TABELA 4. Taxas de mortalidade (por 100000 habitantes) por armas de fogo segundo grupo etário e taxas de variação anual (\%), estado do Rio de Janeiro, Brasil, 1979 a 1992

\begin{tabular}{cccccc}
\hline Ano & $\begin{array}{c}10 \text { a } 14 \\
\text { anos }\end{array}$ & $\begin{array}{c}15 \text { a } 19 \\
\text { anos }\end{array}$ & $\begin{array}{c}20 \text { a } 24 \\
\text { anos }\end{array}$ & $\begin{array}{c}25 \text { a } 29 \\
\text { anos }\end{array}$ & $\begin{array}{c}30 \text { a } 39 \\
\text { anos }\end{array}$ \\
\hline 1979 & 2,2 & 24,4 & 46,1 & 41,6 & 34,2 \\
1980 & 2,6 & 30,7 & 57,9 & 49,4 & 35,6 \\
1981 & 2,1 & 25,9 & 50,0 & 49,5 & 34,6 \\
1982 & 1,9 & 22,5 & 49,3 & 43,1 & 29,6 \\
1983 & 1,5 & 28,4 & 56,0 & 59,8 & 36,5 \\
1984 & 2,6 & 37,0 & 76,7 & 69,9 & 47,5 \\
1985 & 2,4 & 49,5 & 88,1 & 82,2 & 48,7 \\
1986 & 3,7 & 54,8 & 100,2 & 85,8 & 58,3 \\
1987 & 4,9 & 62,5 & 103,9 & 98,2 & 63,2 \\
1988 & 4,2 & 79,3 & 115,2 & 102,6 & 68,5 \\
1989 & 6,6 & 105,7 & 150,6 & 128,5 & 85,3 \\
1990 & 6,0 & 97,7 & 133,9 & 117,3 & 74,1 \\
1991 & 5,8 & 89,1 & 128,0 & 103,5 & 71,2 \\
1992 & 4,2 & 86,9 & 127,3 & 106,6 & 67,5 \\
Taxa de variação & & & & & \\
anual (\%) & $9,9^{\mathrm{a}}$ & $13,6^{\mathrm{a}}$ & $10,0^{\mathrm{a}}$ & $9,1^{\mathrm{a}}$ & $8,0^{\mathrm{a}}$ \\
\hline
\end{tabular}

a Significativo em nível de $5 \%$.

fogo é de grande complexidade e envolve diferentes dimensões. Primeiro, é fundamental compreender que estas mortes, independentemente das circunstâncias de ocorrência, têm sempre um fator comum: o disparo de uma arma (14). O amplo acesso e a disponibilidade de armas entre a população favorecem o crescimento das fatalidades devidas às armas de fogo (15-17).

No estado do Rio de Janeiro, a proliferação das armas envolve dois aspectos de naturezas diversas, embora interligados. $\mathrm{O}$ primeiro refere-se ao crime organizado, que a partir dos anos 80 ficou centralizado no tráfico de drogas e trouxe crescimento substancial na aquisição de armas para o controle dos pontos de tráfico (18). O segundo diz respeito ao número crescente de indivíduos armados. Em busca de segurança, a população, sentindo-se constantemente ameaçada pela escalada da atividade criminal, adquire, cada vez mais, o seu próprio armamento.

Diversos estudos têm demonstrado que o uso indiscriminado de armas não traz nenhum benefício e que, pelo contrário, causa danos e mortes (19). Os cidadãos comuns não têm preparo técnico nem psicológico para usar armas e, não raro, pequenos conflitos terminam em tragédias $(20,21)$. 
TABELA 5. Taxas de mortalidade (por 100000 habitantes) por armas de fogo segundo região de residência e taxas de variação anual (\%), estado do Rio de Janeiro, Brasil, 1979 a 1992

\begin{tabular}{ccccc}
\hline Ano & Capital & $\begin{array}{c}\text { Cinturão } \\
\text { metropolitano }\end{array}$ & Interior & Estado \\
\hline 1979 & 22,3 & 20,4 & 13,7 & 19,7 \\
1980 & 28,1 & 21,6 & 16,2 & 23,3 \\
1981 & 21,6 & 24,4 & 16,0 & 21,2 \\
1982 & 20,0 & 21,8 & 13,2 & 19,0 \\
1983 & 26,8 & 25,0 & 16,1 & 23,7 \\
1984 & 31,9 & 33,1 & 23,7 & 30,3 \\
1985 & 36,8 & 38,2 & 25,0 & 34,5 \\
1986 & 39,1 & 43,3 & 31,3 & 38,6 \\
1987 & 41,5 & 46,5 & 37,2 & 42,0 \\
1988 & 48,2 & 51,3 & 39,4 & 47,1 \\
1989 & 59,0 & 65,0 & 51,2 & 59,0 \\
1990 & 53,8 & 60,9 & 53,0 & 55,9 \\
1991 & 53,1 & 55,3 & 50,7 & 53,1 \\
1992 & 51,8 & 56,2 & 62,1 & 55,6 \\
Taxa de variação anual (\%) & $8,6^{\mathrm{a}}$ & $10,2^{\mathrm{a}}$ & $13,7^{\mathrm{a}}$ & $10,1^{\mathrm{a}}$ \\
\hline
\end{tabular}

a Significativo em nível de $5 \%$.

Segundo dados da polícia civil, a grande maioria das armas de fogo existentes no estado do Rio de Janeiro não é registrada legalmente. Acreditase que o maior volume entre clandestinamente pelo Paraguai e dali chegue ao seu destino pelas estradas, já que não existe fiscalização organizada para prevenir o contrabando de armas. Dada a grande extensão do território brasileiro e do seu litoral, o Brasil oferece muitas perspectivas para este tipo de atividade clandestina, seja pelo ar, por mar ou por terra.

Algumas providências estão sendo tomadas pelo governo federal para controlar o contrabando de armas, tais como a intensificação das ações de fiscalização nas fronteiras, a identificação dos compradores de armas no exterior e a criação de um sistema informatizado, com o registro de todas as armas produzidas, importadas ou vendidas no país. Adicionalmente, um recente projeto de lei tornou o porte ilegal de armas um crime.

Mesmo que a restrição legal ao acesso às armas de fogo produza efeito significativo na redução dos homicídios (22), no âmbito da saúde pública o tratamento da disseminação da violência deve envolver outros componentes além das medidas de controle da proliferação de armas. Todavia, embora o tema da violência tenha ganhado espaço crescente na imprensa e provocado debates na sociedade, ainda são escassos, no Brasil, os projetos de pesquisa interdisciplinares que visam a esclarecer e compreender o âmago da questão.

Introduzidas recentemente aos estudos epidemiológicos, as técnicas de análise estatística espacial muito têm contribuído para a caracterização geográfica dos padrões de morbilidade (23). No caso de detecção de um componente espacial por meio do instrumental estatístico, a análise recai sobre a apreciação de fatores que tornam a distribuição espacial não aleatória e sobre a identificação de áreas de maior interesse para posteriores investiga-

TABELA 6. Taxas de mortalidade (por 100000 habitantes) por armas de fogo e taxas de variação anual (\%) nos grupos etários de 15 a 19 anos e 20 a 24 anos, sexo masculino, segundo região de residência, estado do Rio de Janeiro, Brasil, 1979 a 1992

\begin{tabular}{|c|c|c|c|c|c|c|}
\hline Ano & \multicolumn{3}{|c|}{15 a 19 anos } & \multicolumn{3}{|c|}{20 a 24 anos } \\
\hline 1980 & 80,7 & 55,6 & 24,4 & 129,6 & 116,4 & 60,4 \\
\hline 1981 & 63,0 & 52,5 & 17,4 & 99,8 & 116,4 & 62,1 \\
\hline 1982 & 54,2 & 45,0 & 17,5 & 102,5 & 117,9 & 42,8 \\
\hline 1985 & 127,0 & 97,8 & 33,6 & 189,0 & 200,0 & 96,5 \\
\hline 1986 & 132,1 & 119,9 & 41,3 & 200,1 & 236,1 & 111,0 \\
\hline 1987 & 151,9 & 129,4 & 59,5 & 224,7 & 229,8 & 125,1 \\
\hline 1988 & 190,8 & 177,9 & 56,7 & 255,1 & 244,9 & 138,0 \\
\hline 1989 & 244,8 & 227,7 & 95,3 & 331,1 & 326,9 & 182,5 \\
\hline 1990 & 230,1 & 215,2 & 84,8 & 296,2 & 283,8 & 160,4 \\
\hline 1991 & 218,6 & 179,7 & 70,8 & 276,3 & 277,4 & 162,7 \\
\hline
\end{tabular}


A. 1981

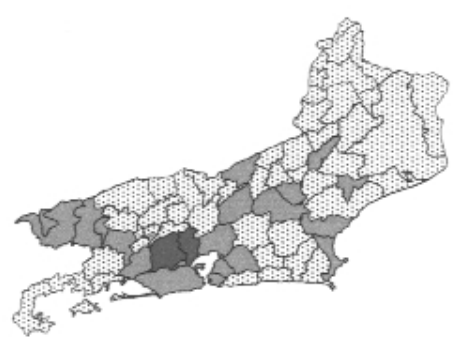

C. 1987

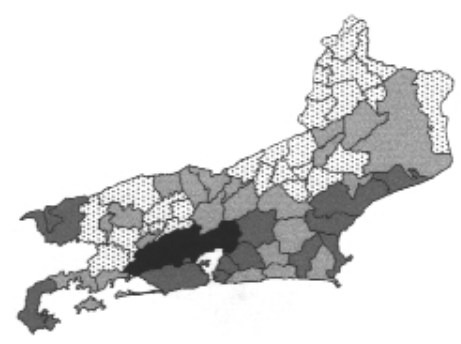

B. 1984

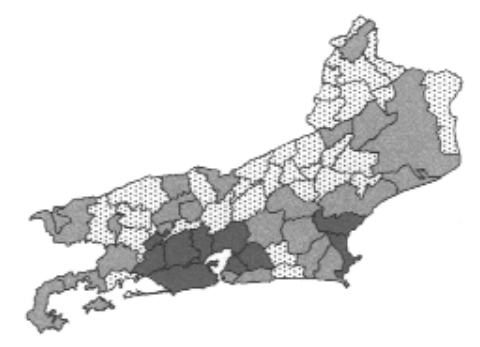

D. 1990

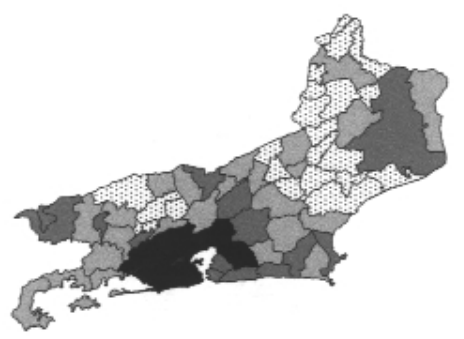

Taxa por armas de fogo (por 100000$)$

0 a 10

10 a 25

25 a 50

50 e mais ções (24). No caso do presente estudo, a análise espacial demonstrou a interiorização de um processo que, tendo iniciado na região metropolitana do estado, se propagou rapidamente para o interior em meados dos anos 80 . Os resultados indicaram, de início, uma direção preferencial de disseminação, ao longo dos municípios situados na costa leste, seguindo o trajeto da rodo- via federal BR-101, que margeia o litoral do estado do Rio de Janeiro. Ao final do período, o alastramento se deu em praticamente todas as direções.

No que se refere à aplicação das técnicas de análise espacial ao estudo da mortalidade por armas de fogo, sabemos que não basta investigar o local de residência para identificar as áreas de maior risco. É preciso, também, inves-

TABELA 7. Coeficientes direcionais de autocorrelação $c$ por período, estado do Rio de Janeiro, Brasil, 1980 a 1991

\begin{tabular}{cccccc}
\hline \multirow{2}{*}{$\begin{array}{c}\text { Direção } \\
(\mathrm{rd})\end{array}$} & $\begin{array}{c}\text { Número } \\
\text { de pares }\end{array}$ & 1980 a 1982 & 1983 a 1985 & 1986 a 1988 & 1989 a 1991 \\
\cline { 3 - 5 } & 58 & $0,5080^{\mathrm{a}}$ & $0,5423^{\mathrm{a}}$ & $0,4168^{\mathrm{a}}$ & $0,3554^{\mathrm{a}}$ \\
$\pi / 8$ & 48 & $0,4201^{\mathrm{a}}$ & 0,6251 & $0,3848^{\mathrm{a}}$ & $0,2389^{\mathrm{a}}$ \\
$\pi / 4$ & 42 & 0,5991 & $0,3795^{\mathrm{a}}$ & $0,2208^{\mathrm{a}}$ & $0,2659^{\mathrm{a}}$ \\
$3 \pi / 8$ & 44 & 0,6217 & 0,6286 & 0,6014 & $0,3786^{\mathrm{a}}$ \\
$\pi / 2$ & 30 & 0,5008 & 0,4232 & 0,4513 & 0,5616 \\
$5 \pi / 8$ & 32 & 0,4280 & 0,5151 & 0,4033 & $0,3428^{\mathrm{a}}$ \\
$3 \pi / 4$ & 36 & 0,6319 & 0,6118 & 0,6226 & $0,3421^{\mathrm{a}}$ \\
$7 \pi / 8$ & 26 & 0,5148 & 0,8442 & 0,6854 & 0,3677 \\
Onidirecional & 316 & $0,5285^{\mathrm{a}}$ & $0,5639^{\mathrm{a}}$ & $0,4591^{\mathrm{a}}$ & $0,3469^{\mathrm{a}}$ \\
\hline
\end{tabular}

a Significativo em nível de 5\%.

tigar o local de ocorrência das mortes. Contudo, no presente trabalho, por motivos operacionais, a análise geográfica se restringiu apenas aos municípios de residência.

A constatação empírica de uma expansão generalizada da criminalidade nos últimos anos, fora da região metropolitana, nega as afirmações corriqueiras de concentração da violência nos bolsões de pobreza das metrópoles brasileiras. Se, por um lado, a falta de controle do contrabando de armas é um agente facilitador, é preciso compreender, por outro lado, por que certos segmentos populacionais são tão vulneráveis, acarretando uma propagação tão rápida da epidemia das mortes por arma de fogo pelo interior do estado.

Até o momento, são conhecidos poucos estudos que tiveram como objeto de investigação a análise geográfica da mortalidade por armas de fogo. Um atlas de mortalidade por causas externas foi elaborado nos Estados Unidos, no período de 1979 a 1987, para fornecer subsídios para estratégias de intervenção através da identifi- 


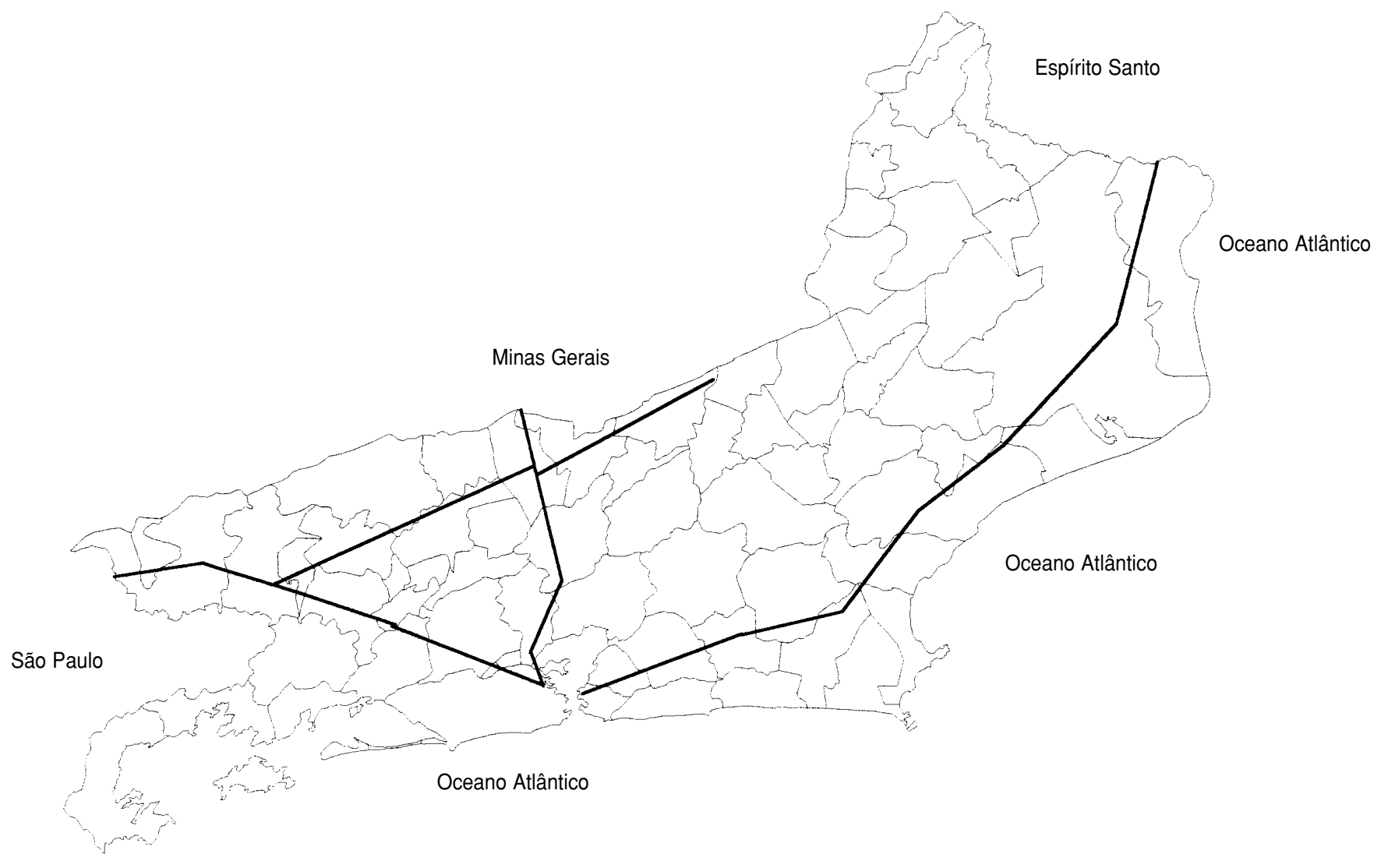

cação das áreas de maior risco (25). Quanto à disseminação espacial do fenômeno, levantamentos feitos na cidade mexicana de Tijuana indicaram uma conexão com a expansão do tráfico de drogas em anos recentes; o aumento do tráfico, por sua vez, é explicado pela vizinhança desta cidade ao estado da Califórnia (26).

As pesquisas realizadas entre grupos sociais específicos têm sugerido uma possível relação entre a freqüência de homicídios e o consumo de drogas (27-31). Porém, os resultados mais conclusivos referem-se à associação entre homicídios e o tráfico de drogas $(32,33)$. Um estudo realizado em Nova Iorque estabeleceu que entre todos os homicídios analisados (um quarto do total de homicídios naquela cidade em 1988), $43 \%$ eram decorrentes do tráfico de drogas (34).

No Brasil, a introdução de crianças e jovens carentes ao tráfico de drogas é um processo facilitado por aparentes vantagens imediatas. A idéia de dinheiro fácil, a ilusão do poder através do porte de arma, a imagem do bandidoherói, a falta de outras oportunidades no mercado de trabalho e a vontade de se expor ao perigo e à aventura atraem fortemente os adolescentes de baixa renda a este tipo de atividade (20).

A facilidade de captação de menores ao tráfico de drogas pode ser interpretada como uma decorrência das desigualdades sociais do país, que aumentaram na última década (35). O perfil brasileiro de concentração de renda se reflete fortemente na exclusão de oportunidades, passando pela ausência de nutrição, educação, assistência médica, habitação e formação profissional adequadas.

Recentemente, comprovou-se a associação entre alguns agravos de saúde e destes com as mortes violentas, assim como com os índices de de- sigualdade social (36-38). Embora os mecanismos desta associação ainda não estejam bem explicados, imaginase que as sociedades que têm grande concentração de renda são também as que não investem em políticas públicas sociais.

Os estudos feitos com jovens em comunidades carentes nos Estados Unidos identificaram a pobreza, o abuso de drogas, a capacitação profissional deficiente dos pais, a ausência de um lar e a falta de integração com seus pares como os principais fatores associados à predisposição do jovem a uma vida de violência e atividade criminal (39).

Todavia, outros fatores podem também explicar o aumento generalizado das mortes por armas de fogo entre a população jovem. Embora não se disponha de dados no Brasil, os resultados de estudos realizados nos Estados Unidos indicam que grande número de jovens vêm tendo práticas compor- 
tamentais que aumentam o seu risco de morrer de maneira violenta e que o fenômeno não se restringe apenas às minorias de alto risco (40). Uma investigação realizada naquele país, em nível nacional, baseada em informações coletadas em escolas durante o ano de 1993, mostrou que mais do que $20 \%$ das crianças entrevistadas tinham portado uma arma durante os 30 dias antecedentes ao inquérito (41). Outras pesquisas, com adolescentes de 9 a 15 anos, em 1992 e 1993, revelaram percentuais elevados de jovens envolvidos com o tráfico de drogas, percentuais estes que chegavam a $15 \%$ na faixa etária de 13 a 15 anos. Indicaram também proporções elevadas de jovens que mencionaram ter sido solicitados a vender drogas ou que manifestaram vontade de se envolver no tráfico (34).

\section{CONCLUSÕES}

Os achados deste estudo demonstraram que o fenômeno do aumento da mortalidade por armas de fogo atinge fortemente os adolescentes de 15 a 19 anos. Este segmento populacional apresentou a maior taxa de aumento da mortalidade por armas de fogo no período estudado, de 13 a $16 \%$ ao ano, conforme a região de residência. Destacaram-se também os adultos jovens, de 20 a 24 anos de idade, do sexo masculino, para quem foram registrados coeficientes muito elevados no Grande Rio, com valores, por vezes, superiores a 300 por 100000 habitantes e cujas taxas anuais de acréscimo se aproximaram de $10 \%$. Exibindo o mesmo comportamento que o grupo de adolescentes, o maior percentual de aumento foi encontrado fora da região metropolitana, no interior do estado. Em situação deveras preocupante estão as crianças de 10 a 14 anos que, apresentando aumento expressivo nos últimos anos, chamam a atenção para um outro aspecto relevante, a propagação das armas de fogo entre grupos cada vez mais jovens.

A análise geográfica demonstrou a proliferação dos óbitos por armas de fogo em toda a extensão do estado do Rio de Janeiro, em uma dinâmica muito veloz de disseminação, que mostrou a grande vulnerabilidade da população ao problema. Acreditase que taxas elevadas de mortalidade por armas de fogo já devam estar sendo encontradas nos municípios que fazem fronteira com o estado ao norte (estados do Espírito Santo e Minas Gerais) e que poderão evoluir rapidamente para outras unidades do país.

A concentração das mortes entre os adolescentes, o aumento expressivo encontrado entre as crianças de 10 a 14 anos e a propagação do processo em ritmo extremamente veloz são especialmente alarmantes e exigem providências imediatas.

A prevenção e o controle da violência devem abordar o problema enfocando desde questões no plano coletivo, tais como a proliferação de armas de fogo, vinculada ao crescimento da atividade criminal, a expansão do narcotráfico e a situação aguda das desigualdades sociais, até questões de ordem individual, como as relações e interações dos jovens com seu ambiente, em nível de família, escola e sociedade. Urge que se coloque em prática estratégias específicas e programas de intervenção de diferentes naturezas, envolvendo a diversidade destes aspectos, para conter esta desastrosa epidemia.

\section{REFERÊNCIAS}

1. Minayo MCS. A violência social sob a perspectiva da saúde pública. Cad Saude Publica 1994:10(Supl 1):7-18.

2. Souza ER. Homicídios no Brasil: o grande vilão da saúde pública na década de 80 . Cad Saude Publica 1994;10(Supl 1):45-60.

3. Reichenheim ME, Werneck GL. Anos potenciais de vida perdidos no Rio de Janeiro, 1990: as mortes violentas em questão. Cad Saude Publica 1994;10(Supl 1):188-198.

4. Szwarcwald CL, Leal MC. Sobrevivência ameaçada dos jovens brasileiros: a dimensão da mortalidade por armas de fogo. Em: Comissão Nacional de População e Desenvolvimento. Jovens acontecendo na trilha das políticas públicas. Brasília: CNPD; 1988.

5. Edelman P, Satcher D. Violence prevention as a public health priority. Health Aff 1993;12: 123-125.

6. Cliff AD, Ord JK. Spatial process: models and applications. London: Pion, 1981.
7. Brasil, Ministério da Saúde. Sub-sistema de informações sobre mortalidade, Brasil, 1979-1993 [CD ROM]. Data/SUS: Brasília; 1996.

8. Fundação Instituto Brasileiro de Geografia e Estatística. Censo demográfico, Brasil 1980. Rio de Janeiro: IBGE; 1983.

9. Fundação Instituto Brasileiro de Geografia e Estatística. Censo Demográfico, Brasil 1991. Rio de Janeiro: IBGE; 1994.

10. MapInfo. Desktop mapping software. New York: MapInfo Corporation, 1994.

11. Yunes J, Rajs D. Tendencia de la mortalidade por causas violentas en la población general y entre los adolescentes y jóvenes de la Región de las Américas. Cad Saude Publica 1994;10 (Supl 1):88-125.

12. U.S. Centers for Disease Control and Prevention. Deaths resulting from firearm and motorvehicle-related injuries-United States 19681991. MMWR Morb Mortal Wkly Rep 1994; 43:37-42.
13. Bourbeau R. Analyse comparative de la mortalité violente dans les pays développés et dans quelques pays en développement durant la période 1985-1989. World Health Stat Q 1993;46:4-33.

14. Chapdelaine A, Samson E, Kimberley MD, Viau L. Firearm-related injuries in Canada: issues for prevention. Can Med Assoc J 1991; 145:1217-1223.

15. Goldberg BW, vonBorstel ER, Dennis LK, Wall E. Firearm injury risk among primary care patients. J Fam Pract 1995;41:158-162.

16. Katcher ML. Firearm injuries among children and adolescents: I) the facts. Wis Med J 1994; 93:511-515.

17. Karlsson T, Isaksson B, Ormstad K. Gunshot fatalities in Stockolm, Sweden with special reference to the use of illegal weapons. J Forensic Sci 1993;38:1409-1421.

18. Minayo MCS. Inequality, violence and ecology in Brazil. Cad Saude Publica 1994;10:241-250. 
19. Hemenway D, Solnick SJ, Azrael DR. Firearm training and storage. J Am Med Assoc 1995; 273:46-50.

20. Kellerman AL, Rivara FP, Rushforth NB, Banton JG, Reay DT, Francisco JT, et al. Gun ownership as a risk factor for homicide in the home. New Engl J Med 1993;329:1084-1091.

21. Mercy JA. Public health policy for preventing violence. Health Aff 1993;12:7-29.

22. Loftin C, McDowall D, Wiersema B, Cottey TJ. Effects of restrictive licensing of handguns on homicide and suicide in the district of Columbia. New Engl J Med 1991;325:1615-20.

23. Marshall RJ. A review of methods for the statistical analysis of spatial patterns of disease. J Royal Stat Soc Series A 1991;154:421-441.

24. Wartemberg D, Greenberg M. Detecting disease clusters: the importance of statistical power. Am J Epidemiol 1990;132(Suppl):156-166.

25. U.S. Centers for Disease Control and Prevention. Injury mortality atlas of the United States 1979-87. MMWR Morb Mortal Wkly Rep 1991;40:846-848

26. Suarez Toriello JE. Problemática social en una ciudad de la frontera: Tijuana - sus causas y sus implicaciones. Em: México, Secretaria de Salud, Consejo Nacional Contra las Adicciones. Centros de Integración Juvenil. Heroína. México: Talleres Gráficos de la Nación; 1989: 57-74.

27. McGonigal MD, Cole J, Schwab CW, Kauder DR, Rotondo MR, Angood PB. Urban firearm deaths: a five-year perspective. J Trauma 1993; 35:532-6.

28. Fendrich M, Mackesy-Amite ME, Goldstein $P$, Spunt M, Brownstein H. Substance involvement among juvenile murderers: comparisons with older offenders based on interviews with prison inmates. Int J Addict 1995;30:1363-1382.

29. Yarvis RM. Patterns of substance abuse and intoxication among murderers. Bull Am Acad Psychiatry Law 1994;22:133-144.

30. Spunt B, Goldstein P, Brownstein H, Frendrich $\mathrm{M}$. The role of marijuana in homicide. Int J Addict 1994;29:195-213.

31. Hanzlick R, Gowitt GT. Cocaine metabolite detection in homicide victims. J Am Med Assoc 1991;256:760-761.

32. MacGregor FE. Estudios sobre la violencia en el Perú. Rev Neuropsiquiatr 1991;54:3-8.

33. Zaluar A, Noronha JC, Albuquerque C. Violência: pobreza ou fraqueza institucional? Cad Saude Publica 1994;10:213-217.

34. Stanton B, Galbraith J. Drug trafficking among African-American early adolescents: prevalence, consequences, and associated behaviors and beliefs. Pediatrics 1994; 93:1029-1043.

35. Sabóia J. Distribuição de renda e pobreza metropolitanas no Brasil. Em: Minayo MCS, organizador. Os limites da exclusão social: meninos e meninas de rua no Brasil. São Paulo: Hucitec; 1993:31-64.

36. Kaplan GA, Pamuck ER, Lynch JW, Cohen $\mathrm{RD}$, Balfour JL. Inequality in income and mor- tality in the United States: analysis of mortality and potential pathways. Br Med J 1996; 312:999-1003.

37. Kennedy BP, Kavachi I, Protow-Stith D. Income distribution and mortality: cross-sectional ecological study of the Robin Hood index in the United States. Br Med J 1996;312:1004-1007.

38. Wallace R. Urban desertification, public health and public order: "planned shrinkage," violent death, substance abuse and AIDS in the Bronx. Social Sci Med 1990;31:801-813.

39. Friday JC. The psychological impact of violence in underserved communities. J Health Care Poor Underserved 1995;6:403-409.

40. Callaham CM, Rivara FP. Urban high school youth and handguns: a school-based survey. J Am Med Assoc 1992;267:3038-3042.

41. Kann L, Warren CW, Harris WA, Collins JL, Douglas KA, Collins ME, et al. Youth risk behavior surveillance: United States, 1993. MMWR Morb Mortal Wkly Rep 1995;44:1-56.

Manuscrito recebido em 3 de janeiro de 1997. Aceito em versão revisada em 22 de abril de 1998.
ABSTRACT Mortality caused by firearms
in the state of Rio de Janeiro,
Brazil: a spatial analysis
Mortality caused by firearms has been increasing at an alarming rate in the state of Rio de Janeiro, Brazil. This study analyzes the gradual evolution of firearm mortality rates in this Brazilian state from 1979 to 1992, according to sex, age, and area of residence (capital city, metropolitan area, or the state's interior), and uses spatial statistical techniques to describe the propagation of this firearm mortality epidemic in time and space.

During the period analyzed, mortality due to firearms showed the greatest increase among 15- to 19-year-old male adolescents, with yearly rates ranging from 13 to $16 \%$, according to area of residence. For children 10 to 14 years of age, mortality caused by firearms increased by $10 \%$ annually in the same period. The highest annual increase occurred in the state's interior. At the beginning of the period studied, dissemination of firearm mortality was observed to follow a definite direction parallel to the federal road that runs along the east coast of the state. Between 1990 and 1992, however, the increase in deaths by firearms spread out in practically every direction. Empirical confirmation of a general expansion of firearm wound mortality contradicts the usual claim that violence is concentrated in areas of extreme poverty within Brazil's largest cities. Programs for prevention and control of this epidemic should focus on its various aspects and take into consideration both collective issues (such as proliferation of firearms among persons involved with international firearm smuggling, increases in criminal activity, expansion of drug trafficking, and exclusion from social opportunities) and personal issues (relationships and interaction of young people with their families, schools, and social environment). 